\title{
Lithium chloride induces mesenchymal-to-epithelial reverting transition in primary colon cancer cell cultures
}

\author{
VALERIA COSTABILE ${ }^{1}$, FRANCESCA DURATURO ${ }^{1}$, PAOLO DELRIO $^{2}$, DANIELA REGA $^{2}$, \\ UGO PACE $^{2}$, RAFFAELLA LICCARDO ${ }^{1}$, GIOVANNI BATTISTA ROSSI ${ }^{3}$, RITA GENESIO ${ }^{1}$, \\ LUCIO NITSCH $^{1}$, PAOLA IZZO ${ }^{1,4}$ and MARINA DE ROSA ${ }^{1}$ \\ ${ }^{1}$ Department of Molecular Medicine and Medical Biotechnology, University of Naples Federico II; \\ ${ }^{2}$ Colorectal Surgical Oncology, and ${ }^{3}$ Endoscopy Unit, Istituto Nazionale per lo Studio e la Cura dei Tumori, \\ 'Fondazione Giovanni Pascale' IRCCS, I-80131 Naples; ${ }^{4}$ CEINGE Biotecnologie Avanzate, I-80145 Naples, Italy
}

Received October 26, 2014; Accepted December 18, 2014

DOI: 10.3892/ijo.2015.2911

\begin{abstract}
Epithelial-to-mesenchymal transition (EMT) confers stem cell-like phenotype and more motile properties to carcinoma cells. During EMT, the expression of E-cadherin decreases, resulting in loss of cell-cell adhesion and increased migration. Expression of Twist1 and other pleiotropic transcription factors, such as Snail, is known to activate EMT. We established primary colon cancer cell cultures from samples of operated patients and validated cultures by cytogenetic and molecular biology approaches. Western blot assay, quantitative real-time PCR and immunofluorescence were performed to investigate the expression of E-cadherin, vimentin, $\beta$-catenin, cytokeratin-20 and -18, Twist1, Snail, CD44, cyclooxygenase-2 (COX2), Sox 2, Oct4 and Nanog. Moreover, cell differentiation was induced by incubation with $\mathrm{LiCl}$-containing medium for 10 days. We observed that these primary colorectal cancer (CRC) cells lost expression of the E-cadherin epithelial marker, which was instead expressed in cancer and normal colon mucosa of the same patient, while overexpressed vimentin (mesenchymal marker), Twist1, Snail (EMT markers) and COX2. Cytokeratin-18 was expressed both in tissues and cell cultures. Expression of stem cell markers, such as CD44, Oct4 and Nanog, were also observed. Following differentiation with
\end{abstract}

Correspondence to: Dr Marina De Rosa, Department of Molecular Medicine and Medical Biotechnology, University of Naples Federico II, via S. Pansini 5, I-80131 Naples, Italy

E-mail: marina.derosa@unina.it

Abbreviations: EMT, epithelial-to-mesenchymal transition; MET, mesenchymal-to-epithelial transition; CRC, colorectal cancer; CIN, chromosomal instability; MIN, microsatellite instability; MMR, mismatch repair genes; HM, healthy mucosa; TT, tumor tissue; CIMP, CpG island methylator phenotype

Key words: colorectal cancer, primary colon cancer cell cultures, epithelial-to-mesenchymal transition, mesenchymal-to-epithelial transition, stem cell-like phenotype, GSK $3 \beta$ inhibition the glycogen synthase kinase $3 \beta$ (GSK3 $\beta$ ) inhibitor $\mathrm{LiCl}$, the cells began to express E-cadherin and, at once, Twist1 and Snail expression was strongly downregulated, suggesting a MET-reverting process. In conclusion, we established primary colon mesenchymal cancer cell cultures expressing mesenchymal and epithelial biomarkers together with high level of EMT transcription factors. We propose that they could represent a good model for studying EMT and its reverting mechanism, the mesenchymal-to-epithelial transition (MET). Our observation indicates that $\mathrm{LiCl}$, a GSK3 $\beta$ inhibitor, induces MET in vitro, suggesting that $\mathrm{LiCl}$ and GSK3 $\beta$ could represent, respectively, interesting drug, and target for CRC therapy.

\section{Introduction}

Colorectal cancer (CRC) is the second cause of cancer deaths in Europe and the third in the United States, with $>1$ million new cases estimated every year. Death occurs because of metastasis to other organs (1).

Epithelial cells from gastrointestinal trait acquire sequential genetic and epigenetic mutations in specific oncogene and/or tumor suppressor genes, during colorectal adenocarcinoma development, conferring them a selective advantage on proliferation and self-renewal (2). Thus, normal epithelium becomes hyperproliferative mucosa and subsequently gives rise to a benign adenoma that progressively evolves into carcinoma and, subsequently, generates metastasis (3-5).

Loss of genomic integrity facilitates the accumulation of multiple mutations during the development of CRC. Chromosomal instability (CIN), microsatellite instability (MIN), aberrant DNA methylation and DNA repair defects are all mechanisms involved in colorectal epithelial cell transformation (6).

At molecular level CRCs are a very heterogeneous group of diseases showing several alterated molecular signaling pathways, such as Wnt/APC/ $\beta$-catenin,PI3K/Akt/glycogen synthase kinase $3 \beta$ (GSK3 $\beta$ ), TGF $\beta / \mathrm{SMAD}$, NF- $\kappa \mathrm{B}$ or mismatch repair genes (MMR). These alterations confer individual susceptibility to cancer, and are responsible for responsiveness or resistance to antitumor agents (7). 
Wnt/ $/$-catenin pathway is the most frequently dysfunctional signaling in sporadic CRC. When Wnt ligand, a secreted glycoprotein, binds to its frizzled receptors, the multifunctional kinase GSK $3 \beta$ is inactivated and $\beta$-catenin, that acts both as E-cadherin cell-cell adhesion protein and as a transcriptional activator, is stabilized, accumulated in the cytoplasm and finally translocates into nucleus. Here it interacts with members of the lymphoid enhancer factor (LEF)/T-cell factor (TCF) and activates specific target genes. In the absence of Wnt signal, casein kinase 1 (CK1) and the APC/Axin/GSK3 $\beta$ complex, target $\beta$-catenin for ubiquitination and proteasomal degradation by its phosphorylation (8).

GSK3 $\beta$ is a multifunctional serine/threonine kinase and an important regulator of cell survival that may act as anti- or pro-apoptotic, in a cell-specific manner. Its activity is regulated by site-specific phosphorylation. Full activity of GSK3 $\beta$ generally requires phosphorylation at tyrosine 216 (Tyr216), whereas phosphorylation at serine 9 (Ser9) inhibits its activity. It has been demonstrated that activation of PI3K and PKC inhibits GSK $3 \beta$ activity by stimulating its Ser9 phosphorylation (9).

GSK3 $\beta$ also regulates NF- $\kappa$ B activity, a transcription factor that plays a role in many physiological and pathophysiological processes, including immune responses, inflammation, cell proliferation, survival and differentiation. In colon and pancreatic cancer cells, NF- $\kappa \mathrm{B}$ is positively regulated by GSK $3 \beta$ and its activation confers a selective growth advantage on these cells, so acting as a tumor promoter. The molecular mechanisms underlying GSK3 $3 / \mathrm{NF}-\kappa \mathrm{B}$ interaction remain to be further investigated $(10,11)$. More than 100 proteins, playing a role in a wide spectrum of cellular processes, are substrates of GSK $3 \beta$, among which, $\beta$-catenin and NF- $\kappa B$ inhibitor I $\mathrm{KB}$ are the most well-known. Genes upregulated by $\beta$-catenin/TCF/LEF and/or NF- $\mathrm{kB}$ include proto-oncogenes, such as $c-M y c$ and cyclin-Dl, and genes regulating cell invasion/migration, such as Snail, CD44 and MMP-7 (12).

Recently, it has been suggested that epithelial-to-mesenchymal transition (EMT) could be a common biological mechanism that could represent a good target for therapeutic intervention. EMT consists in an essential phenotypic conversion of epithelial cells into cells with mesenchymal phenotype. It is a reversible process that often occurs during embryonic development and tissue remodeling and also plays a critical role in early events occurring in invasion and metastasis of many types of cancer, including CRC (13). EMT regulation is orchestrated by a group of transcription factors, including Snail, Slug, ZEB1 and Twist, but tumor microenvironment also plays a part into phenotypic conversion through different signals, such as TGF $\beta$, EGF, Wnt and Notch $(14,16)$.

During EMT epithelial cells lose their E-cadherin expression, that specifically guarantees the epithelial phenotype, destroy their intercellular adhesion, acquire mesenchymal characteristics and increase migratory and invasive properties. Furthermore, the EMT program induces stem cell-specific gene expression, thus promoting self-renewal capability (14-16).

One of the main problems in cancer treatment is drug resistance, responsible for relapses in many tumors and the failure of medical treatments in metastatic disease. Probably, both chemotherapy and radiation therapy too often miss the opportunity to kill a part of a tumor cell subpopulation, such as CSC and CSC-like cells.
We aimed to realize a tissue biobank from patients affected by CRC and to establish primary cell cultures with the main purpose of studying EMT and its reverting mechanism, the mesenchymal-to-epithelial transition (MET) in CRC, in vitro. We also investigated GSK3 $\beta$ inhibition as therapeutic target of CRC.

\section{Materials and methods}

Patients. Blood samples, normal colorectal mucosa and CRC tissues were obtained from patients with sporadic colon cancer operated at the Istituto Nazionale dei Tumori in Naples (Italy) and tissues were frozen in liquid nitrogen. Primary cell cultures were also established from some of these CRC patients.

Samples from all subjects who participated in the study were collected after being granted authorization from the Comitato Etico per le Attività Biomediche 'Carlo Romano' of the University of Naples Federico II, with protocol no. 120/10. Such authorization is given only once the study has received ethics approval, and the participants informed and written consent has been obtained.

Cell cultures. Samples of CRC from CRC patients were washed overnight at $4^{\circ} \mathrm{C}$ in PBS containing $300 \mathrm{U} / \mathrm{ml}$ penicillin, $300 \mu \mathrm{g} / \mathrm{ml}$ streptomycin, and $2.5 \mu \mathrm{g} / \mathrm{ml}$ amphotericin B (all from Gibco-BRL, Karlsruhe, Germany), finely minced with scissors (tissue pieces of $\sim 30 \mathrm{~mm}^{3}$ ) and digested in $2 \mathrm{ml} 0.1 \%$ collagenase II (Boehringer Mannheim, Mannheim, Germany) in DMEM/FBS- $10 \%$ for $1 \mathrm{~h}$ at $37^{\circ} \mathrm{C}, 5 \% \mathrm{CO}_{2}$. The cell suspension was then collected by centrifugation, washed twice with serum-free DMEM medium, and subsequently cultured in DMEM/F12-10\% FBS medium (1:1), $100 \mathrm{U} / \mathrm{ml}$ penicillin, $100 \mu \mathrm{g} / \mathrm{ml}$ streptomycin, and $2.5 \mu \mathrm{g} / \mathrm{ml}$ amphotericin B (all from Gibco-BRL). CRC cells were selected by differential sedimentation, cultured on plates, and incubated with $\mathrm{LiCl}$ (30 mmol/l) for 1 and $24 \mathrm{~h}$ as well as 10 days. For in vitro differentiation, DMEM/F12-5\% FBS medium $30 \mathrm{mM} \mathrm{LiCl}$ with was used. These primary colon cancer cells were then cultured as spheres in serum-free stem cell medium and low-adhesion plates, as described by Kreso and O'Brien (17), for $\sim 60$ days, disgregated six times every 10 days.

Cytogenetic analysis. Metaphase chromosome analysis was performed on cell cultures from CRC patients, using high resolution G-banding (550 bands) according to standard procedures. Multicolor-FISH (M-FISH) was carried out using MetaSystems' 24XCyte color kit (MetaSystems GmbH, Altlussheim, Germany).

FISH analysis was performed using whole chromosome painting (WCP) probes for chromosomes 20 and 22 and locus-specific DiGeorge probe mixture (MetaSystems $\mathrm{GmbH}$ ) that contains a SpectrumOrange probe located at 22q11.2 and a SpectrumGreen LSI probe that maps at 22q13.3 region and subtelomeric probes for the $\mathrm{p}$ (green) and $\mathrm{q}$ (red) arms of chromosomes 20.

Multicolor chromosome banding (MCB) was performed using the multicolor banding DNA probe kit based on microdissection derived region-specific libraries for chromosome 22 (MetaSystems $\mathrm{GmbH}$ ) according to standard protocols (18). 
Table I. Oligonucleotide sequences.

\begin{tabular}{lll}
\hline Oligonucleotides & \multicolumn{1}{c}{ Primers } & \\
\hline Cytokeratin 18 & F: 5'-AGACTGGAGCCATTACTTC-3' & Accession nos. \\
& R: 5'-GCTCTGTCTCATACTTGACTC-3' & [NM_199187.1; start +441] \\
Cytokeratin 20 & F: 5'-CTGCAAATTGATAATGCTAA-3' & [NM_199187.1; start +563] \\
& R: 5'-GGTCATCAAAGACCTTATTC-3' & [XM_005277792.1; start +485] \\
B-Raf & F: 5'-TGCTTGCTCTGATAGGAAAATGAGA-3' & [XM_005277792.1; start +586] \\
& R: 5'-CTCAGCAGCATCTCAGGGCC-3' & [NC_018918.2; start +140387483] \\
E-cadherin & F: 5'-TCCTGGGCAGAGTGAATTTT-3' & [NC_018918.2; start +140387250] \\
& R: 5'-CCGTAGAGGCCTTTGACTG-3' & [NM_004360.3; start +276] \\
Snail 1 & F: 5'-GCGAGCTGCAGGACTCTAAT-3' & [NM_004360.3; start +381] \\
Twist1 & R: 5'-TCCCAGATGAGCATTGGCAG-3' & [NM_005985.3; start +143] \\
& F: 5'-CCTTCTCGGTCTGGAGGAT-3' & [NM_005985.3; start +269] \\
Vimentin & R: 5'-TCCTTCTCTGGAAACAATGACA-3' & [NM_000474; start +908] \\
& F: 5'-TCTGGATTCACTCCCTCTGG-3' & [NM_000474; start +1004] \\
GUS & R: 5'-GGTCATCGTGATGCTGAGAA-3' & [NM_003380; start +1694] \\
& F: 5'-GAAAATATGTGGTTGGAGAGCTCATT-3' & [NM_003380; start +178 \\
\hline
\end{tabular}

GUS, glucuronidase.

FISH experiments were performed on metaphase spreads and fluorescent images were analysed using a fluorescence microscope (Axio Imager.Z1 mot; Carl Zeiss Microscopy, LLC, Thornwood, NY, USA) with ISIS software imaging system (MetaSystems $\mathrm{GmbH}$ ) for image capturing and processing.

RER assay. The MSI status was confirmed with a fluorescent multiplex system including six mononucleotide repeats (BAT-25, BAT-26, BAT-40, NR21, NR24, and TGF $\beta R I I)$ and four dinucleotide repeats (D2S123, D5S346, D17S250, and D18S58) as described earlier (19), using the CC-MSI kit (AB Analitica s.r.l., Padova, Italy), according to the manufacturer's instructions. PCR products were analysed by capillary electrophoresis analysis using an ABI Prism 3130 Genetic Analyzer (Applied Biosystems, Inc., Foster City, CA, USA).

RT-PCR analysis. Total RNA was extracted from colorectal tissues of CRC patients, using QIAzol Reagent (Qiagen, Hilden, Germany), after homogenization and cDNA was synthesized with $1 \mu \mathrm{g}$ of total RNA, $500 \mathrm{ng}$ of random hexamers, and $1 \mu 1$ SuperScript III Reverse Transcriptase (Invitrogen Life Technologies, Carlsbad, CA, USA), in the presence of $4 \mu \mathrm{l}$ 5X RT buffer, $1 \mu$ DTT $(0.1 \mathrm{M})$ and $1 \mathrm{mM}$ dNTPs, after DNase incubation (Invitrogen Life Technologies). The reaction was run for $50 \mathrm{~min}$ at $42^{\circ} \mathrm{C}$ in a $20 \mu \mathrm{l}$ reaction volume, heated to $70^{\circ} \mathrm{C}$ for $15 \mathrm{~min}$ and quickly chilled on ice. One microliter of the cDNA was amplified by RT-PCR for CTK20 and 18 as well as E-cadherin messengers, using primer pairs shown in Table I.

All oligonucleotides were obtained with Primer-BLAST Software (http://www.ncbi.nlm.nih.gov/tools/primer-blast/).

Real-time PCR quantification analysis. Real-time PCR quantification analysis was performed for Twist1, Snail and cyclooxygenase-2 (COX2) messengers using $0.5 \mu \mathrm{l}$ of the cDNA and primer pairs shown in Table I.

Relative expression was calculated with the comparative $\mathrm{Ct}$ method and normalized against the $\mathrm{Ct}$ of glucuronidase (GUS) mRNA. The quantitative real-time assays were performed using the Bio-Rad iCycler iQ Real-Time PCR Detection System (Bio-Rad Laboratories, Inc., Hercules, CA, USA) as previously described $(20,21)$, using healthy mucosa (HM) as a calibrator to measure the relative expression.

Western blot assay. Total proteins were extracted from HM of colon and colon tumor tissue (TT) of affected patients and from primary colon cancer cultures, using QIAzol Reagent (Qiagen) following the manufacturer's instructions. Concentrations were determined by using a protein assay kit adopting bovine serum albumin standards, according to the manufacturer's instructions (Bio-Rad Laboratories, Inc.). A total of $30 \mu \mathrm{g}$ of proteins were separated by SDS-polyacrylamide gel electrophoresis and blots were prepared on a Amersham Hybond-ECL nitrocellulose membrane (Amersham Pharmacia Biotech, Inc./ GE Healthcare Bio-Sciences Corp., Piscataway, NJ, USA). The primary antibody against $\beta$-catenin, E-cadherin (monoclonal, rabbit anti-human; no. 3195), CD44 (monoclonal, mouse anti-human; no. 5640), Snail (monoclonal, rabbit anti-human; no. 3879) and vimentin (monoclonal, rabbit anti-human; no. 5741) was from Cell Signaling Technology, Inc. (Beverly, MA, USA). The antibody against actin (polyclonal, rabbit anti-human; no. sc-1615) was from Santa Cruz Biotechnology, Inc. (Santa Cruz, CA, USA). The membrane was probed with a secondary antibody against peroxidase-conjugated rabbit or goat immunoglobulin $\mathrm{G}$, and immunoreactive bands were detected using the enhanced chemiluminescence Immobilon Western HRP Substrate (Millipore, Billerica, MA, USA). 
A

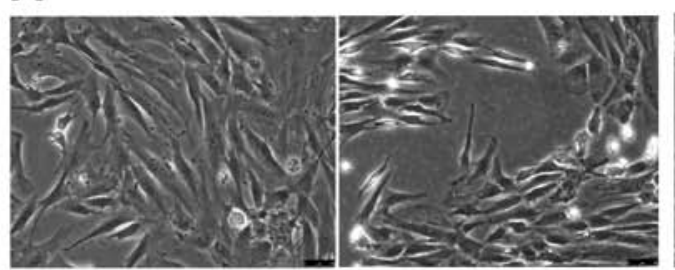

C

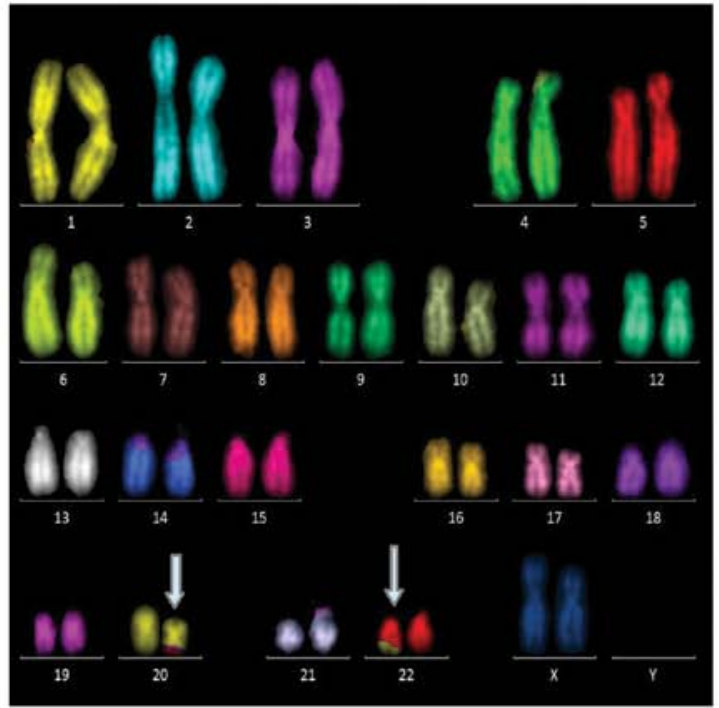

B
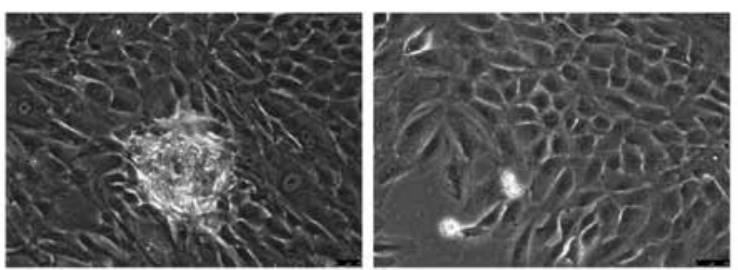

D

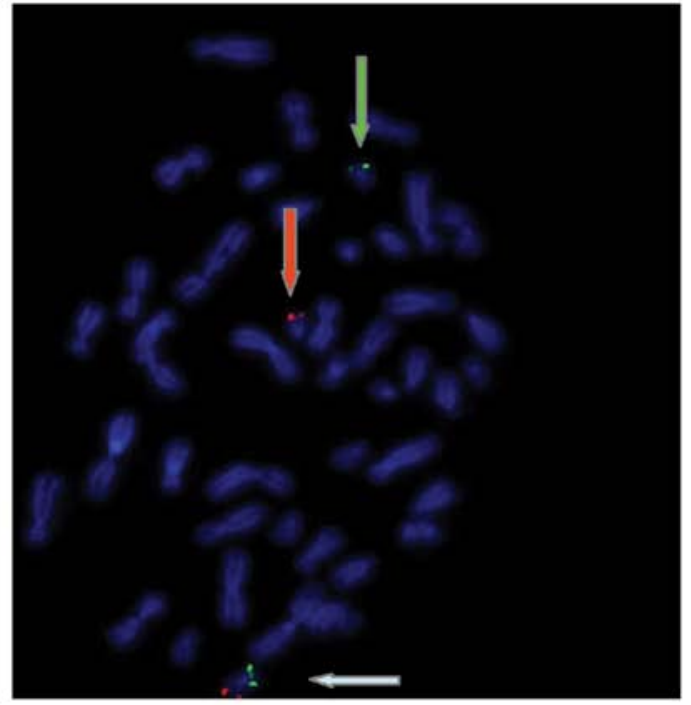

E

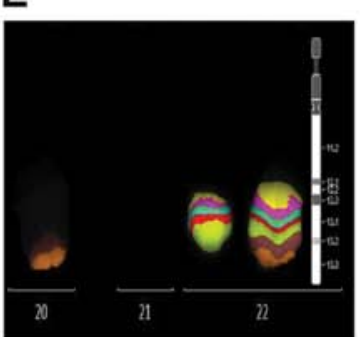

F

$120 \quad 140$

$140 \quad 160$

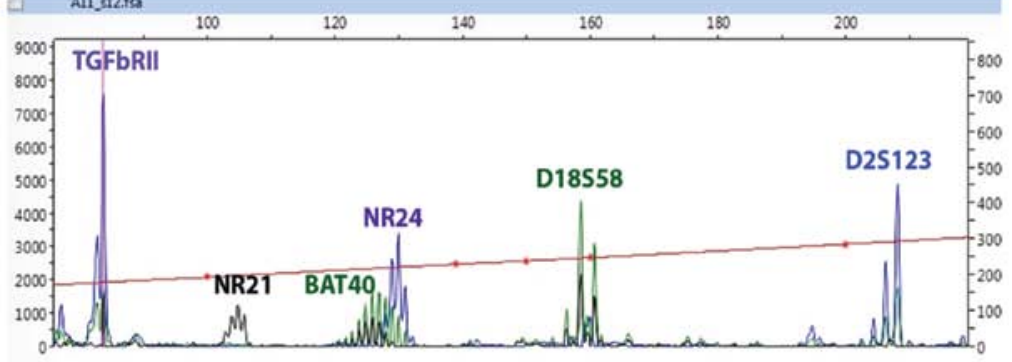

G
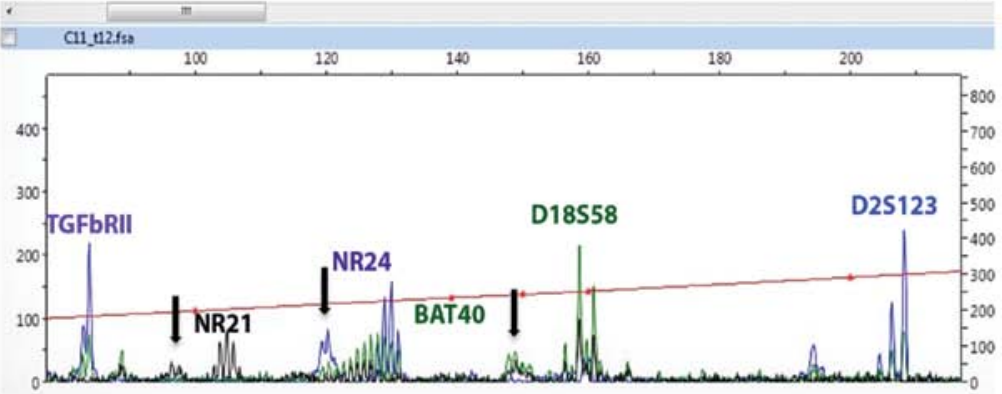

Figure 1. Molecular biology and cytogenetic validation of primary colon cultures. (A) T88 and (B) T93 cell culture images in a bright field with x20 magnification (scale bar, $50 \mu \mathrm{m}$ ). (C-E) Karyotype analysis of T93 cell cultures; (C) multicolor-FISH (M-FISH) showing a reciprocal translocation between chromosomes 20 and 22; (E) high resolution multicolor chromosome banding (MCB) image of the normal chromosome 22 compared to the rearranged chromosome 22; (D) FISH with subtelomeric probes for the $\mathrm{p}$ (green) and $q$ (red) arms of chromosome 20. (F and G) Microsatellite instability (MIN) assay of T88 cell culture performed on DNA from (F) peripheral blood cells and (G) tumor tissue (TT). Black arrows indicate altered microsatellites.

Immunofluorescence. The primary cancer cells were seeded and grown on glass coverslips $24 \mathrm{~h}$ prior to the experiments. After fixation with $4 \%$ paraformaldehyde (PFA)-PBS for 10 min, cells were permeabilized in $0.1 \%$ Triton X-100 in PBS and sequentially blocked with $10 \%$ bovine serum albumin for $45 \mathrm{~min}$. Following the overnight incubation with primary antibody specific for cytokeratin (pan) (no. 18-0059, 1:100 dilution; Zymed Laboratories, Inc., San Francisco, CA, USA),
E-cadherin (no. 3195, 1:100 dilution), Nanog (polyclonal, rabbit anti-human, no. 3580, 1:100 dilution), Sox 2 (monoclonal, mouse anti-human, no. 4900, 1:100 dilution), Oct4 (monoclonal, rabbit anti-human, no. 2840, 1:100 dilution) and Snail (no. 3879, 1:100 dilution), and/or $2 \mathrm{~h}$ incubation with primary antibody specific for vimentin (no. 5741, 1:200 dilution) and CD44 (no. 5640, 1:800 dilution) (all from Cell Signaling Technology, Inc.). Cells were further incubated with appropriate secondary antibodies 
A

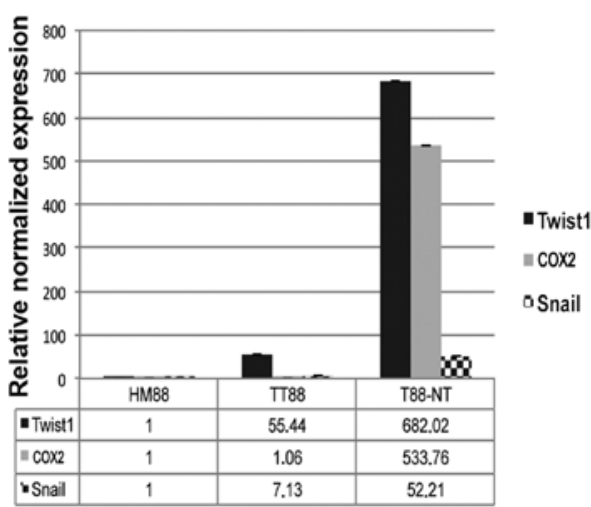

C

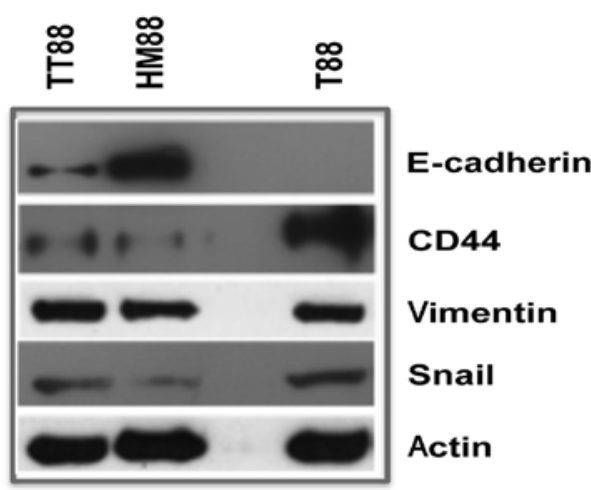

E

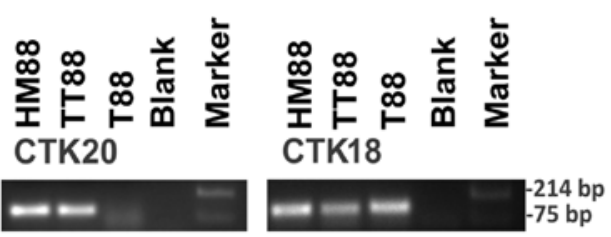

B

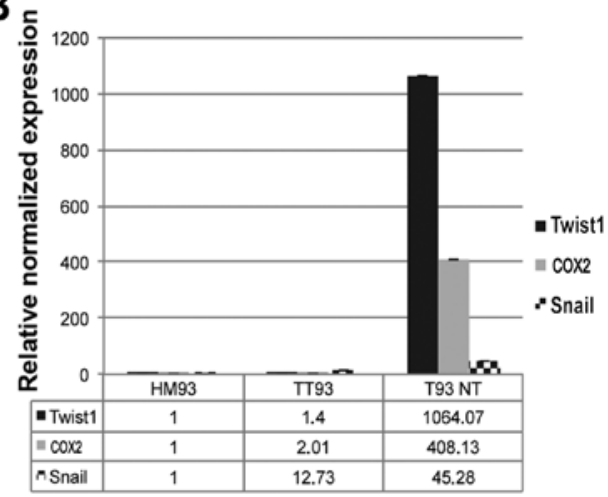

D

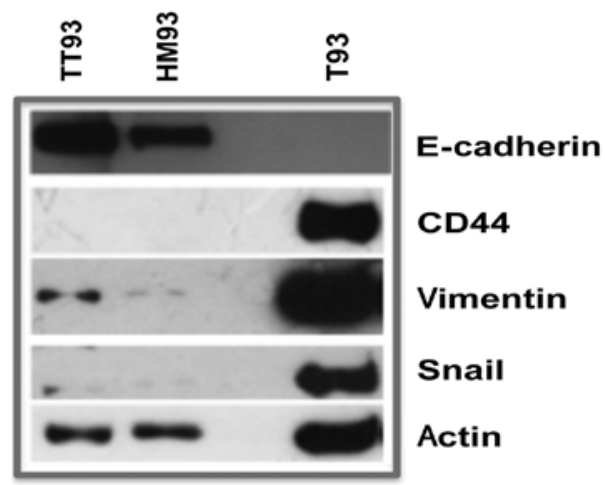

F

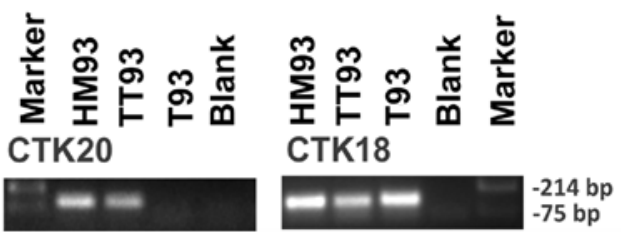

Figure 2. Protein and RNA expression analysis of T88 and T93 primary cell cultures. (A-B) Real-time RT-PCR analysis of Twist1, Snail and cyclooxygenase-2 (COX2), performed on healthy mucosa (HM), tumor tissue (TT), untreated tumor cell cultures (T) from patient (A) no. 88 and (B) no. 93. (C-D) Western blot assay of E-cadherin, CD44, vimentin, Snail and actin performed with $30 \mu \mathrm{g}$ of proteins extracted from HM, TT and untreated T from patient (C) no. 88 and (D) no. 93. (E-F) RT-PCR analysis of cytokeratin 20 and 18 performed on cDNA from HM, TT and untreated T from patient (E) no. 88 and (F) no. 93.

and DAPI for nuclear labeling. The negative controls without primary antibodies were also included, while no obvious staining was observed. Immunofluorescence was visualized under a fluorescence microscope and image-captured.

\section{Results}

Stabilisation of primary colon cancer cell cultures and tumor status validation. We realized a tissue biobank by sampling pairs of healthy colorectal mucosa and its matched CRC tissues from patients with sporadic and hereditary CRC, frozen in liquid nitrogen. From the same CRC tissues we established primary CRC cell cultures. For molecular investigation, we chose two different cultures: one mesenchymal-like (T88) (Fig. 1A) and the other more epithelial-like (T93) (Fig. 1B). According to TNM staging, classification of these two tumors was T3N1 and T4N2, for tumor 88 and 93, respectively. First of all, we validated the cancer status of our cultures using molecular biology and cytogenetic tecniques.
Karyotyping was performed on both cell cultures and cytogenetic analyses were interpreted at a resolution level of 550 bands. Only the T93 cells showed a female karyotype with a reciprocal translocation between the $q$ arm of chromosome 20 and the $\mathrm{q}$ arm of chromosome 22, but no numerical aberrations were found (Fig. 1C). The high resolution MCB image of the normal chromosome 22 compared to the rearranged chromosome 22 shows that the 22 q13.2qter region of chromosome 22 was translocated to chromosome 20, while the region of chromosome 20 translocated includes the region $20 \mathrm{q} 13$ to 20 qter (Fig. 1E). FISH with subtelomeric probes for the $\mathrm{p}$ (green) and $\mathrm{q}$ (red) arms of chromosomes 20 shows that region 20q13 was translocated into chromosome 22 (Fig. 1D).

The complete chromosomal characterisation according to ISCN 2013 (22) was as follows: 46,XX,t(20;22)(q13;q13)(20pter $\rightarrow 20$ q13.2::22q13.2 $\rightarrow$ 22qter:22pter $\rightarrow 22$ q13.1::20q13.3 $\rightarrow$ 20qter).

MSI analysis was performed on DNA extracted from TT and pheripheral blood sample of patient no. 88, resulted negative for CIN phenotype, who was found to have an MSI-high 

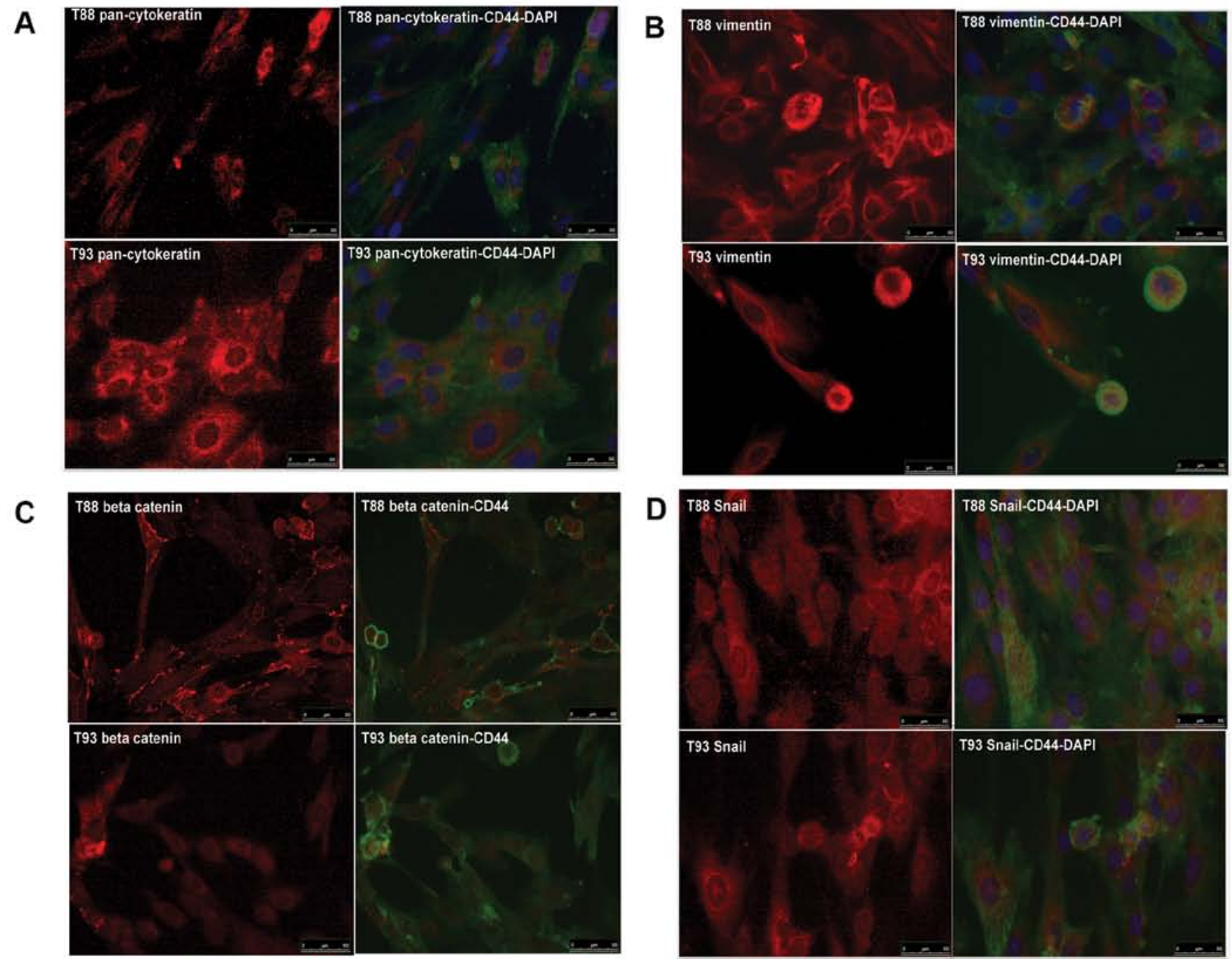

$\mathbf{E}$
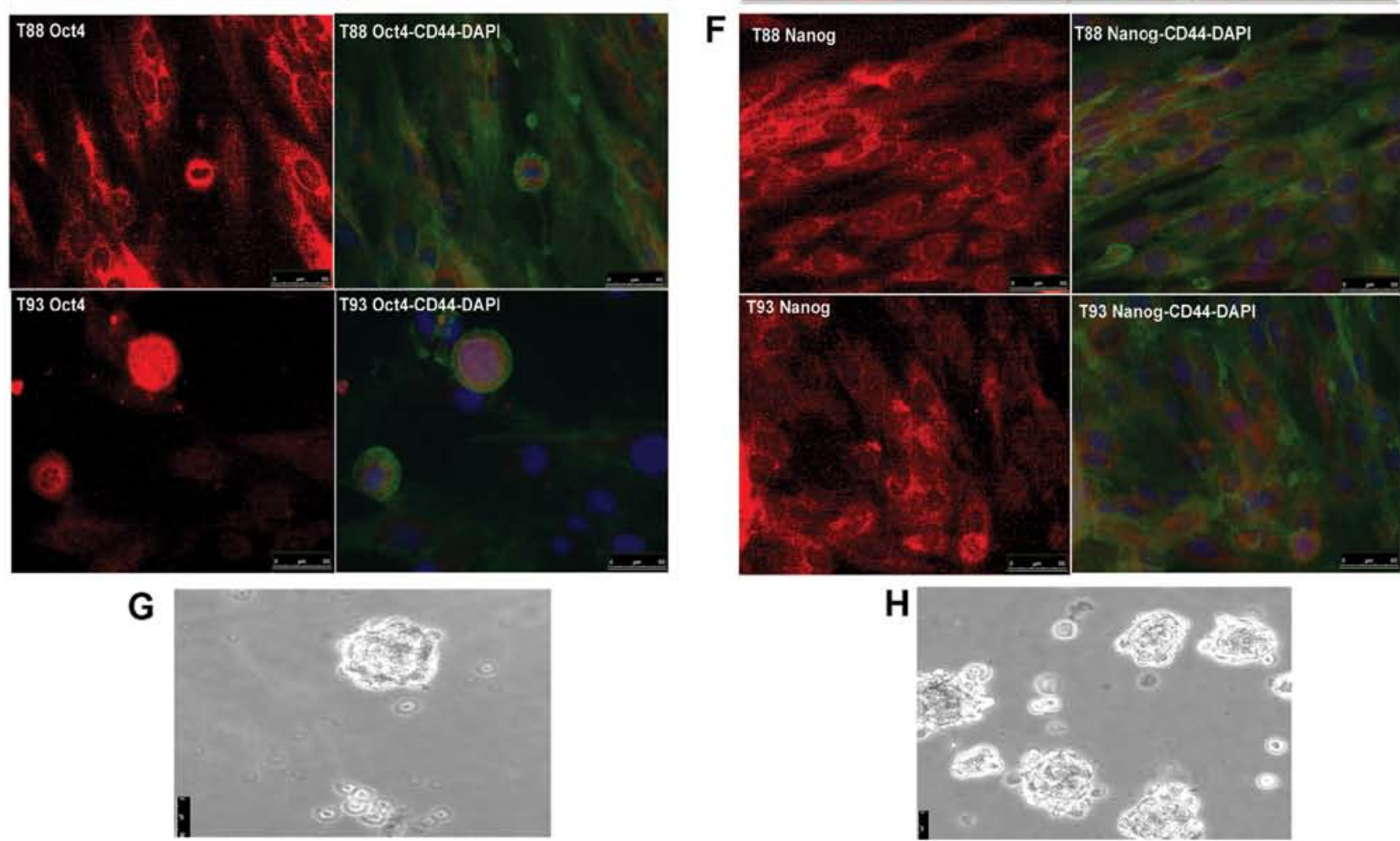

Figure 3. T88 and T93 primary cell cultures express mesenchymal, epithelial-to-mesenchymal transition (EMT) and stem cell characteristic. Immunofluorescence analysis performed on T88 and T93 cells of (A) cytokeratin (pan)/CD44, (B) vimentin/CD44, (C) $\beta$-catenin/CD44, (D) Snail/CD44, (E) Oct4/CD44, (F) Nanog/CD44 and (G and H) colon cancer spheres grown from T88 and T93 cells. Images in bright field. Magnification, x20 (scale bar, $50 \mu \mathrm{m}$ ). 
(MSI-H) status, with instability at mononucleotide markers NR21, BAT-40 and NR24 (Fig. 1F and G).

Cell cultures express mesenchymal and epithelial markers, EMT transcription factors and stemness markers. We have characterized T88 and T93 primary colon cancer cell cultures for epithelial and mesenchymal markers, by using RT-PCR, real-time PCR, western blot and immunofluorescent analysis.

As shown in Fig. 2A and B, Twist1, Snail and COX2 messengers were mainly upregulated in both cell cultures when investigated by real-time RT-PCR. GUS mRNA was used as calibrator in all real-time quantification assays and each HM of colon was adopted as target sample for relative quantification.

Furthermore, E-cadherin protein expression was downregulated in T88 and upregulated in T93 TTs, while completely absent in cell cultures. On the other hand, vimentin and Snail were upregulated in TTs, compared to normal colon mucosa of each patient but highly expressed in cell cultures, when analyzed by western blot assay. CD44, instead, was upregulated in both cell cultures, furthermore it was upregulated in TT from patient no. 88 but it gave no hybridization signal in tissues from patient no. 93, in our experimental conditions (Fig. 2C and D).

Moreover, HM of colon, colon cancer tissues and cell cultures expressed cytokeratin 18, whereas only healthy and cancer mucosa expressed cytokeratin 20, when analyzed by RT-PCR (Fig. 2E and F).

To clarify if all the cells of these cultures expressed mesenchymal markers, EMT-TF and epithelial markers or, alternatively, were a heterogeneous cell population, we performed an immunofluorescence assay using CD44, Snail, vimentin, pan-cytokeratin, Oct4, Nanog and Sox 2 antibody. As shown in Fig. 3, all the cells were positive for all the analyzed proteins except for Sox 2 protein, whose antibody did not give any signal in our experimental condition (data not shown). CD44 was mainly localized at membrane level (Fig. 3A-F), while pan-cytokeratine and vimentin were localized at the cytoskeleton level (Fig. 3A and B) and, as expected, $\beta$-catenin was localized at level of membrane, cytoplasm and nucleus (Fig. 3C). Snail, Oct4 and Nanog showed a turnover between nucleus and cytoplasm (Fig. 3D-F). Furthermore, Oct4 and Nanog were mostly expressed at the level of cancer cell spheres and clones (data not shown). They were also able to grow as a sphere in a conditioned medium without serum and in low adhesion condition (Fig. 3G and $\mathrm{H}$ )

LiCl incubation of primary colon cancer cell culture induces MET and differentiation in vitro. Following incubation with the GSK3 $\beta$ inhibitor $\mathrm{LiCl}$, cells differentiated, as shown in Fig. 4A, B, E and F and $\beta$-catenin, like CD44, become mainly localized at membrane level, as shown by immunofluorescence assay (Fig. 4C, D, G and H). Twist1, Snail, COX2 and CD44 expression was downregulated (Fig. 5A, B, E and F) and cells, originally negative for E-cadherin, started to express this epithelial marker both at RNA (Fig. 5C and D) and protein level (Fig. 5E and F), suggesting a mesenchymal-to-epithelial reverting transition process. The T88-untreated cells were more mesenchymal-like than the T93 cells, and did not express E-cadherin. On the other hand, the T93-untreated cells, that showed more epithelial-like morphology, had low level of E-cadherin transcription (Fig. 5C and D).

When analyzed by western blot assay, Snail and CD44 gave no signal after 10 days of $\mathrm{LiCl}$ incubation in $\mathrm{T} 93$ cells, while they strongly decreased in T88 cells (Fig. 5E and F). Interestingly, $\beta$-catenin and vimentin showed the opposite trend in response to GSK3 $\beta$ inhibition (Fig. 5E and F). After 10 days of $\mathrm{LiCl}$ incubation, expression of vimentin mRNA and protein strongly decreased in T93 cells (Fig. 5F and H) while they increased in T88 cells (Fig. 5E and G). On the other hand, in the same conditions, $\beta$-catenin was upregulated in T93 cells and downregulated in T88 cells (Fig. 5E and F).

\section{Discussion}

We have set up a protocol to isolate and establish primary CRC cell cultures highly enriched in mesenchymal cancer cells derived by an EMT process from epithelial cells. To our knowledge, this is the first primary CRC cell culture isolated from CRC patients expressing mesenchymal and epithelial biomarkers together with high level of EMT transcription factors.

The T88 and T93 cultures were further analysed. T88 cells appeared more mesenchymal-like than T93 cells, that instead showed a more epithelial-like morphology. The cancer nature of these cells was confirmed and validated by MSI and kariotype analysis.

MSI, CpG island methylator phenotype (CIMP) and CIN play a significant role in CRC (6).

CIN occurs in $\sim 60 \%$ of $\mathrm{CRC}$ and concerns different cellular phenomena chacterised by the presence of abnormal chromosome number or complement (23).

MSI consists in variations in the number of repetitive units in each microsatellite. It is caused by failure of the DNA mismatch repair system to repair errors occurring during replication. These mistakes are usually corrected by the MMR which include hMLH1, hMSH2 and hMSH6 (24). There are two well-established MSI phenotypes, namely MSI-H and MSI-low (MSI-L or MSS). We found that T88 cells showed MIN, whereas T93 cells were carriers of a 46,XX,t $(20 ; 22)$ (q13;q13) translocation in $95 \%$ of metaphases. Both regions on chromosome 20 and 22, involved in this translocation, are reported to be alterated in sporadic CRC (25-27). We concluded that both cell cultures were highly enriched with colon cancer cells.

Furthermore, we showed that these cells expressed mesenchymal markers, EMT-TF and epithelial markers together, therefore they have to be considered mesenchymal colon cancer cells that have undergone EMT from epithelial adenocarcinoma cells. In agreement with each morphological phenotype, T88 cells, that were more mesenchymal-like than T93 cells, did not express E-cadherin, not even at mRNA level and showed a higher increment in Twist1 mRNA expression in TT compared to T93 cells, that showed more epithelial-like morphology and had a low level of E-cadherin transcription (Fig. 5C and D).

According to literature data $(28,29)$, these cells express some stem cell markers, such as Oct4 and Nanog and are able to grow as spheres in a conditioned medium without serum and in low adhesion condition. 

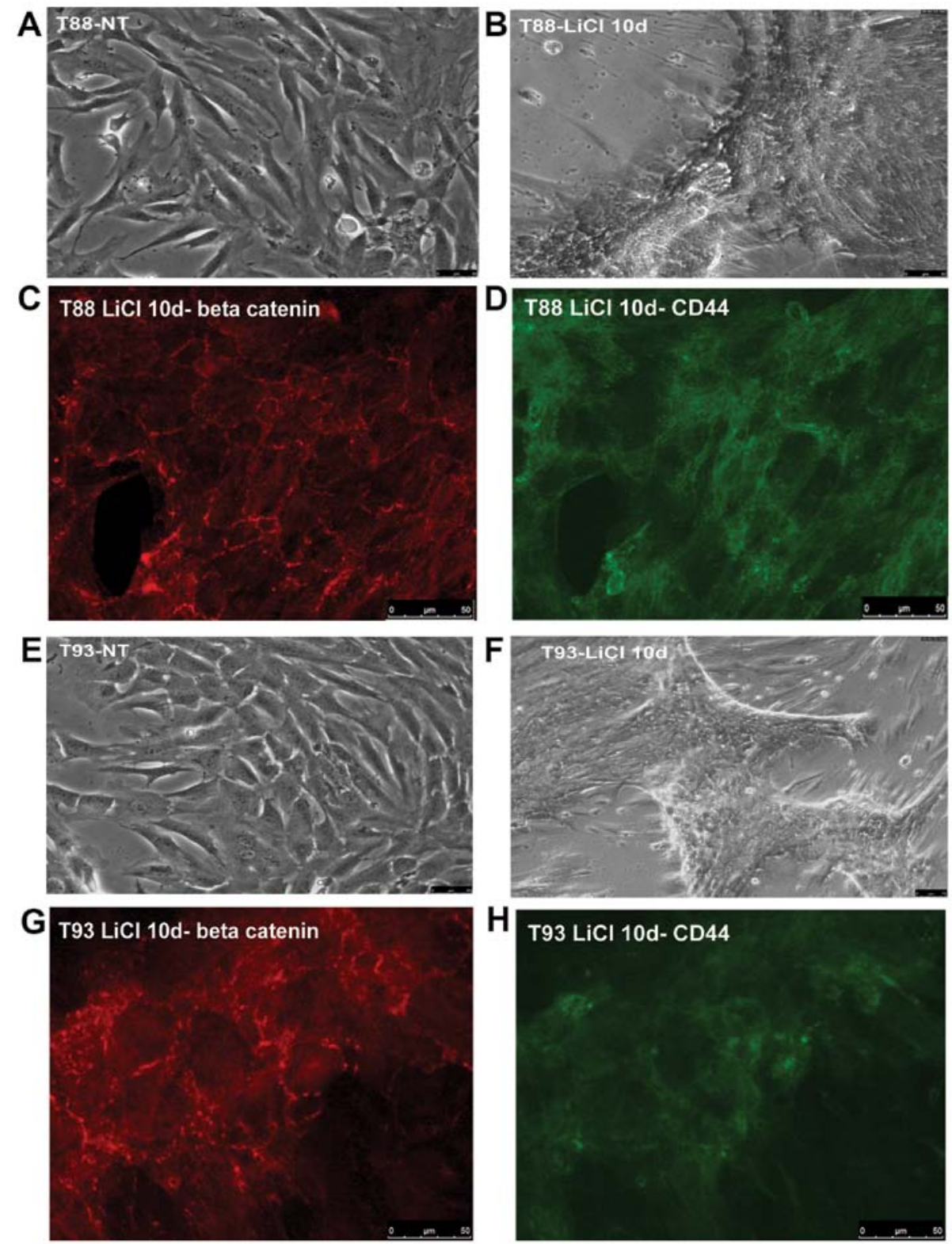

Figure 4. LiCl induces in vitro differentiation. Cell culture images in bright field of (A) T88 and (E) T93 at $\mathrm{T}_{0}$ and (B and F) after 10 days of LiCl incubation. Immunofluorescence assay of (C and G) $\beta$-catenin and (D and H) CD44 on differentiated cells. Magnification, x20 (scale bar, $50 \mu \mathrm{m}$ ).

We were able to induce a mesenchymal-to-epithelial reverting transition under incubation with the GSK3 $\beta$ inhibitor $\mathrm{LiCl}$. $\mathrm{LiCl}$ is the most studied between GSK3 $\beta$ inhibitors and exerts its action through two well-known mechanisms. As a direct inhibitor, lithium is a competitive inhibitor of the $\mathrm{Mg}^{2+}$, which results in inhibition of the activity of this enzyme. On the other end, it also acts in an indirect manner, causing a large increase in the phosphorylation of Ser9 of GSK3 $\beta$ (30).

In our experience, after 10 days of $\mathrm{LiCl}$ incubation, cells were able to transcribe E-cadherin; Twist1 and Snail mRNA was strongly downregulated as well as CD44 and Snail protein, when investigated by western blot assay. Interestingly, COX2 mRNA was highly overexpressed in both cell cultures, compared to their matched colon mucosae and it was also highly downregulated after $\mathrm{LiCl}$ incubation. According to these results, literature data indicate a strong association between $\mathrm{COX} 2$ expression and cancer progression and metastasis (31).
As stated in the Introduction, GSK3 $\beta$ can act as pro-apoptotic signal negatively regulating Wnt signaling, by controlling the degradation of $\beta$-catenin. On the other hand, it positively regulates NF- $\kappa \mathrm{B}$ pathway by mediating the degradation of IкB, a central inhibitor of NF- $\mathrm{KB}$, so inducing an anti-apoptotic responce (32).

In colon and pancreatic cancer cells, GSK $3 \beta$ activates a proliferative signal by activation of NF- $\kappa \mathrm{B}$, and inactivation of GSK3 $\beta$ inhibits NF- $\kappa B$ activity $(33,34)$.

Interestingly, Snail, CD44, G3BP2, and YAP1 are targets of Wnt5A, a gene involved in invasion and metastasis of many cancers, that is regulated by NF- $\kappa B$ signaling pathway $(35,36)$.

E-cadherin and its transcriptional repressor Snail orchestrate a functional cross-regulation between Wnt and NF- $\mathrm{\kappa B}$ pathways during EMT. Expression of Snail promotes E-cadherin downregulation destroying epithelial organization and inducing mesenchymal phenotype of epithelial cells, that 
A

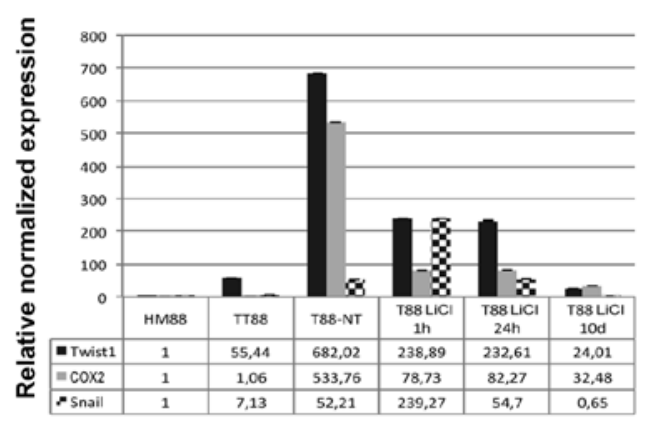

C

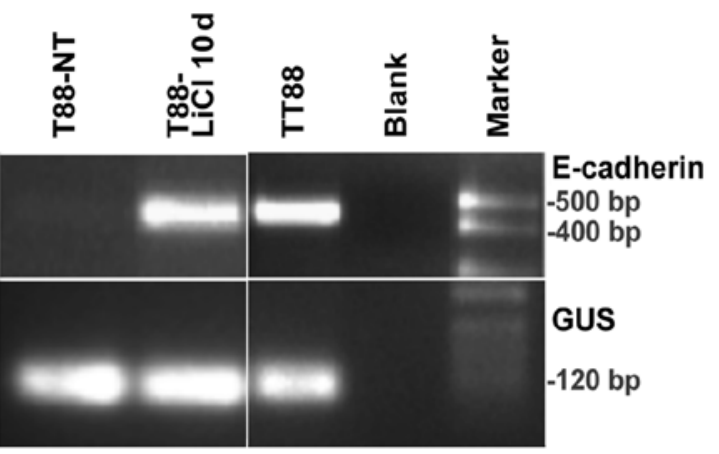

E
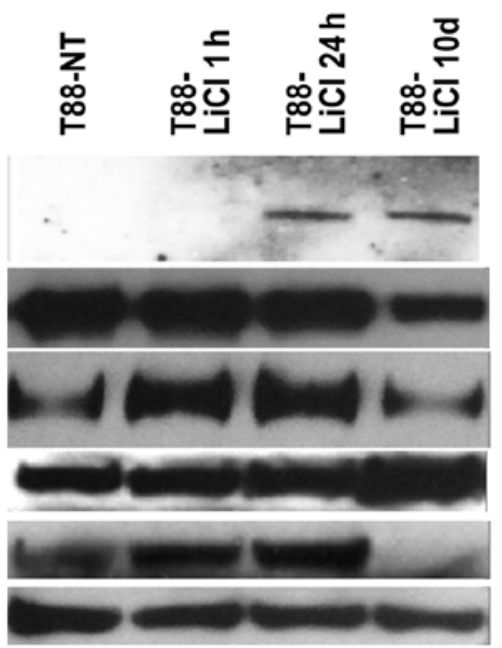

E-cadherin

$\beta$-catenin

CD-44

Vimentin

Snail

Actin

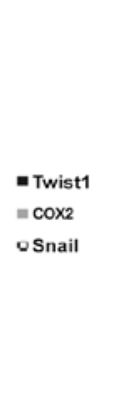

D
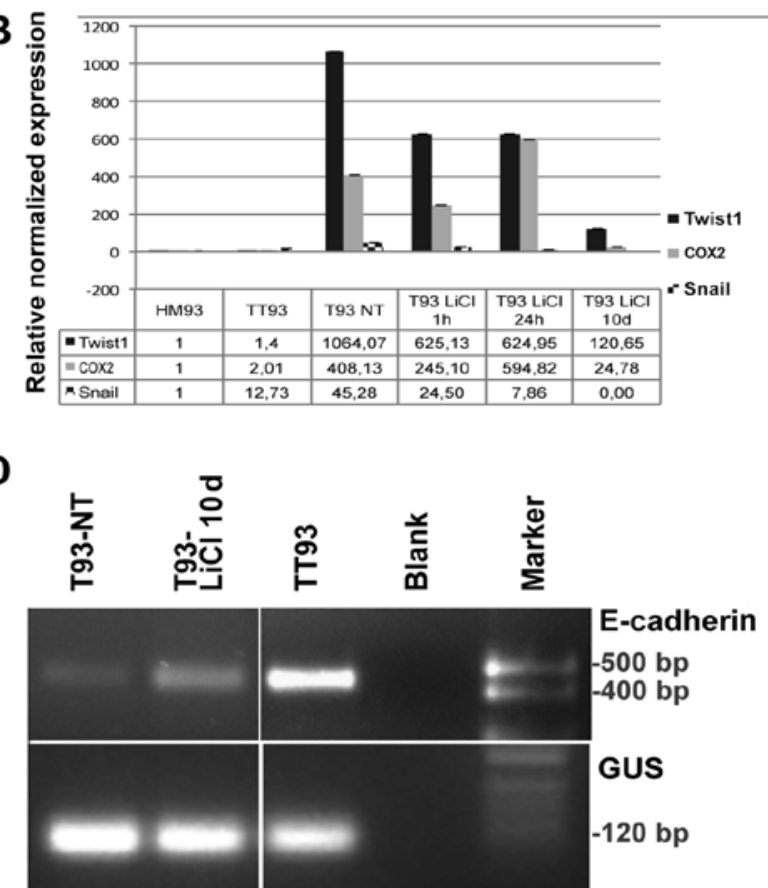

F

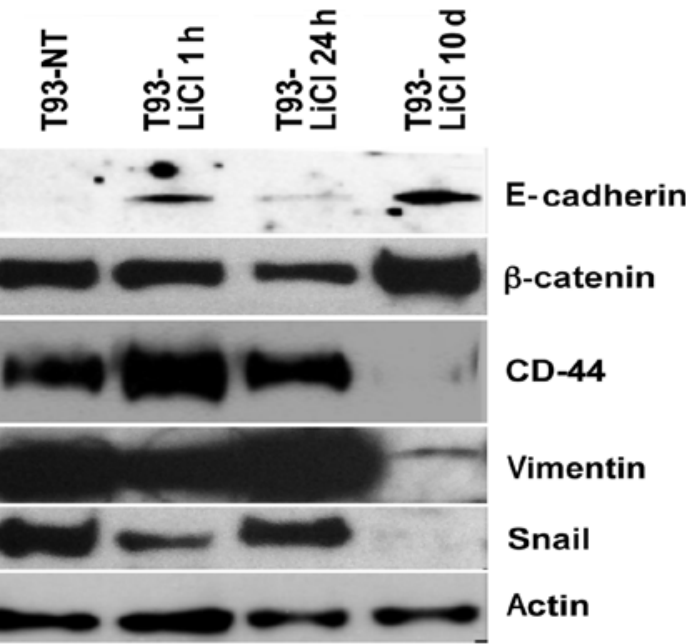

H

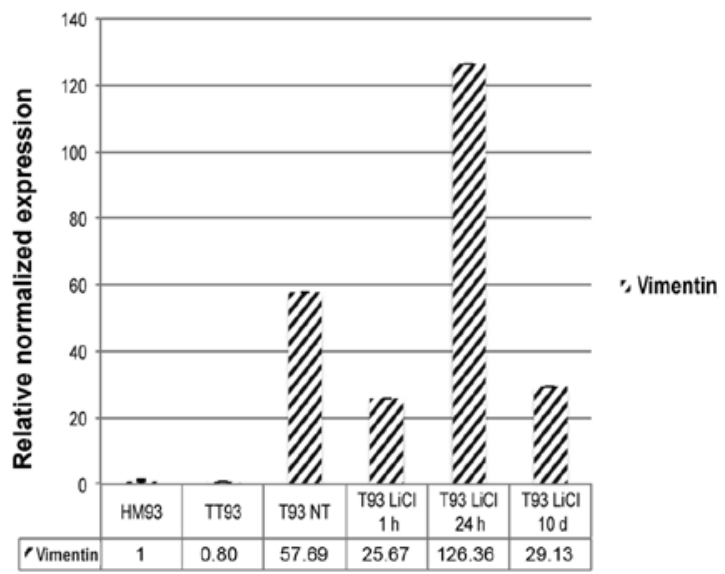

Figure 5. LiCl incubation of primary cancer cell cultures induces mesenchymal-to-epithelial transition (MET) in vitro. (A and B) Real-time RT-PCR analysis of Twist1, Snail and cyclooxygenase-2 (COX2) performed on healthy mucosa (HM), tumor tissue (TT), untreated tumor cell cultures (T), and tumor cells after 1 and $24 \mathrm{~h}$ as well as 10 days of $\mathrm{LiCl}$ incubation from patient (A) no. 88 and (B) no. 93. (C and D) RT-PCR analysis of E-cadherin performed on cDNA from HM, TT and untreated T from patient (C) no. 88 and (D) no. 93. (E and F) Western blot assay of $\beta$-catenin, E-cadherin, CD44, vimentin, Snail and actin performed on proteins extracted from untreated T, and tumor cells after 1 and $24 \mathrm{~h}$ as well as 10 days of LiCl incubation of (E) T88 and (F) T93 cells. ( $\mathrm{G}$ and H) Real-time RT-PCR analysis of vimentin performed on HM, TT, untreated T, and tumor cells after 1 and $24 \mathrm{~h}$ as well as 10 days of LiCl incubation from patient $(\mathrm{G})$ no. 88 and $(\mathrm{H})$ no. 93 . 
takes place together with upregulation of mesenchymal genes expression. E-cadherin and other cell adhesion components directly bound both $\beta$-catenin and NF- $\kappa \mathrm{B}$, sequestering them into adherens junctions and therefore preventing the transcription of target genes $(37,38)$.

As recently described (39), we suggest that GSK3 $\beta$ inhibition could represent a good strategy targeting the cross-regulation between these two pathways and may be a promising direction for future cancer therapy that needs to be better elucidated.

Cell culture models obviously do not mimic the complex interaction that are found in the intestinal mucosa and a functional change in the cell cultures cannot tell us whether there will be the same effect in vitro. Nevertheless cell cultures are used in an attempt to make complex systems more simple by isolating certain functions and investigate them in detail. From this point of view, we speculate that our cell culture could represent an interesting model to further investigate the molecular biology of mesenchymal CRC cells, clinical relevance of EMT in human $\mathrm{CRC}$ and the molecular basis of pharmacological resistance and metastasis.

In conclusion, our preliminary data suggest that EMT could represent a common mechanism among very different colon cancer types and $\mathrm{LiCl}$, a GSK3 $\beta$ inhibitor, induces MET in both cell types despite their genotype, suggesting that $\mathrm{LiCl}$ and GSK $3 \beta$ could represent, respectively, interesting drug and target for CRC therapy. However, it seems to be more efficient in T93 cells, characterized by a CIN phenotype, than in T88 cells, that showed MIN phenotype, even if the first one was a more advanced stage carcinoma. Obviously, larger population studies and further investigations will be necessary to confirm our results.

\section{Acknowledgements}

This study has been supported by the agreement 2010-2012 between CEINGE and Campania Regional Authority; POR Campania FSE 2007-2013.

\section{References}

1. Ewing I, Hurley JJ, Josephides E and Millar A: The molecular genetics of colorectal cancer. Frontline Gastroenterol 5: 26-30, 2014.

2. Hanahan D and Weinberg RA: Hallmarks of cancer: the next generation. Cell 144: 646-674, 2011.

3. Fearon ER and Vogelstein B: A genetic model for colorectal tumorigenesis. Cell 61: 759-767, 1990.

4. Yeung AT, Patel BB, Li XM, Seeholzer SH, Coudry RA, Cooper HS, Bellacosa A, Boman BM, Zhang T, Litwin S, Ross EA, Conrad P, Crowell JA, Kopelovich L and Knudson A: One-hit effects in cancer: altered proteome of morphologically normal colon crypts in familial adenomatous polyposis. Cancer Res 68: 7579-7586, 2008.

5. Phelps RA, Chidester S, Dehghanizadeh S, Phelps J, Sandoval IT, Rai K, Broadbent T, Sarkar S, Burt RW and Jones DA: A two-step model for colon adenoma initiation and progression caused by APC loss. Cell 137: 623-634, 2009.

6. Sideris M and Papagrigoriadis S: Molecular biomarkers and classification models in the evaluation of the prognosis of colorectal cancer. Anticancer Res 34: 2061-2068, 2014.

7. Fearon ER: Molecular genetics of colorectal cancer. Annu Rev Pathol 6: 479-507, 2011.

8. Moon RT, Kohn AD, De Ferrari GV and Kaykas A: WNT and beta-catenin signalling: diseases and therapies. Nat Rev Genet 5: 691-701, 2004
9. McCubrey JA, Steelman LS, Bertrand FE, Davis NM, Sokolosky M, Abrams SL, Montalto G, D'Assoro AB, Libra M, Nicoletti F, Maestro R, Basecke J, Rakus D, Gizak A, Demidenko ZN, Cocco L, Martelli AM and Cervello M: GSK-3 as potential target for therapeutic intervention in cancer. Oncotarget 5: 2881-2911, 2014.

10. Hoesel B and Schmid JA: The complexity of NF- $\mathrm{kB}$ signaling in inflammation and cancer. Mol Cancer 12: 86, 2013.

11. Li H, Huang K, Liu X, Liu J, Lu X, Tao K, Wang G and Wang J: Lithium chloride suppresses colorectal cancer cell survival and proliferation through ROS/GSK- $3 \beta / \mathrm{NF}-\kappa \mathrm{B}$ signaling pathway. Oxid Med Cell Longev 2014: 241864, 2014.

12. Mishra R: Glycogen synthase kinase 3 beta: can it be a target for oral cancer. Mol Cancer 9: 144, 2010.

13. Loboda A, Nebozhyn MV, Watters JW, Buser CA, Shaw PM, Huang PS, Van't Veer L, Tollenaar RA, Jackson DB, Agrawal D, Dai $\mathrm{H}$ and Yeatman TJ: EMT is the dominant program in human colon cancer. BMC Med Genomics 4: 9, 2011.

14. De Craene B and Berx G: Regulatory networks defining EMT during cancer initiation and progression. Nat Rev Cancer 13: 97-110, 2013

15. Batlle E, Sancho E, Francí C, Domínguez D, Monfar M, Baulida J and García De Herreros A: The transcription factor snail is a repressor of E-cadherin gene expression in epithelial tumor cells. Nat Cell Biol 2: 84-89, 2000.

16. Cano A, Pérez-Moreno MA, Rodrigo I, Locascio A, Blanco MJ, del Barrio MG, Portillo F and Nieto MA: The transcription factor snail controls epithelial-mesenchymal transitions by repressing E-cadherin expression. Nat Cell Biol 2: 76-83, 2000.

17. Kreso A and O'Brien CA: Colon cancer stem cells. Curr Protoc Stem Cell Biol Chapter 3: Unit 3.1, 2008.

18. Liehr T, Weise A, Heller A, Starke H, Mrasek K, Kuechler A, Weier HU and Claussen U: Multicolor chromosome banding (MCB) with YAC/BAC-based probes and region-specific microdissection DNA libraries. Cytogenet Genome Res 97: 43-50, 2002.

19. Duraturo F, Cavallo A, Liccardo R, Cudia B, De Rosa M, Diana G and Izzo P: Contribution of large genomic rearrangements in Italian Lynch syndrome patients: characterization of a novel alu-mediated deletion. Biomed Res Int 2013: 219897, 2013.

20. Galatola M, Miele E, Strisciuglio C, Paparo L, Rega D, Delrio P, Duraturo F, Martinelli M, Rossi GB, Staiano A, Izzo P and De Rosa M: Synergistic effect of interleukin-10-receptor variants in a case of early-onset ulcerative colitis. World J Gastroenterol 19: 8659-8670, 2013.

21. Galatola M, Paparo L, Duraturo F, Turano M, Rossi GB, Izzo P and De Rosa M: Beta catenin and cytokine pathway dysregulation in patients with manifestations of the 'PTEN hamartoma tumor syndrome' BMC Med Genet 13: 28, 2012.

22. Shaffer LG, McGowan-Jordan J and Schmid M (eds): ISCN 2013: An International System for Human Cytogenetic Nomenclature. 1st edition. S. Karger AG, Basel, pp140, 2013.

23. Alberici P and Fodde R: The role of the APC tumor suppressor in chromosomal instability. Genome Dyn 1: 149-170, 2006.

24. Sameer AS, Nissar S and Fatima K: Mismatch repair pathway: molecules, functions, and role in colorectal carcinogenesis. Eur J Cancer Prev 23: 246-257, 2014.

25. Therkildsen C, Jönsson G, Dominguez-Valentin M, Nissen A, Rambech E, Halvarsson B, Bernstein I, Borg K and Nilbert M: Gain of chromosomal region $20 \mathrm{q}$ and loss of 18 discriminates between Lynch syndrome and familial colorectal cancer. Eur J Cancer 49: 1226-1235, 2013.

26. Castellví-Bel S, Castells A, Johnstone CN, Piñol V, Pellisé M, Elizalde JI, Romo N, Rustgi AK and Piqué JM: Evaluation of PARVG located on 22q13 as a candidate tumor suppressor gene for colorectal and breast cancer. Cancer Genet Cytogenet 144: 80-82, 2003.

27. Zheng HT, Peng ZH, Zhou CZ, Li DP, Wang ZW, Qiu GQ and He L: Detailed deletion mapping of loss of heterozygosity on $22 q 13$ in sporadic colorectal cancer. World J Gastroenterol 11: 1668-1672, 2005.

28. Li X, Pei D and Zheng H: Transitions between epithelial and mesenchymal states during cell fate conversions. Protein Cell 5: 580-591, 2014.

29. Mani SA, Guo W, Liao MJ, Eaton EN, Ayyanan A, Zhou AY, Brooks M, Reinhard F, Zhang CC, Shipitsin M, Campbell LL, Polyak K, Brisken C, Yang J and Weinberg RA: The epithelialmesenchymal transition generates cells with properties of stem cells. Cell 133: 704-715, 2008.

30. Jope RS: Lithium and GSK-3: one inhibitor, two inhibitory actions, multiple outcomes. Trends Pharmacol Sci 24: 441-443, 2003. 
31. Elzagheid A, Emaetig F, Alkikhia L, Buhmeida A, Syrjänen K, El-Faitori O, Latto M, Collan Y and Pyrhönen S: High cyclooxygenase-2 expression is associated with advanced stages in colorectal cancer. Anticancer Res 33: 3137-3143, 2013.

32. Beurel E and Jope RS: The paradoxical pro- and anti-apoptotic actions of GSK3 in the intrinsic and extrinsic apoptosis signaling pathways. Prog Neurobiol 79: 173-189, 2006.

33. Shakoori A, Ougolkov A, Yu ZW, Zhang B, Modarressi MH, Billadeau DD, Mai M, Takahashi Y and Minamoto T: Deregulated GSK3beta activity in colorectal cancer: its association with tumor cell survival and proliferation. Biochem Biophys Res Commun 334: 1365-1373, 2005.

34. Shakoori A, Mai W, Miyashita K, Yasumoto K, Takahashi Y, Ooi A, Kawakami K and Minamoto T: Inhibition of GSK-3 beta activity attenuates proliferation of human colon cancer cells in rodents. Cancer Sci 98: 1388-1393, 2007.

35. Du Q and Geller DA: Cross-regulation between Wnt and NF- $\kappa B$ signaling pathways. For Immunopathol Dis Therap 1: 155-181, 2010.
36. Katoh M and Katoh M: Transcriptional mechanisms of WNT5A based on NF- $\mathrm{B}$, Hedgehog, TGF $\beta$, and Notch signaling cascades. Int J Mol Med 23: 763-769, 2009.

37. Conacci-Sorrell M, Zhurinsky J and Ben-Ze'ev A: The cadherincatenin adhesion system in signaling and cancer. J Clin Invest 109: 987-991, 2002.

38. Solanas G, Porta-de-la-Riva M, Agusti C, Casagolda D, Sánchez-Aguilera F, Larriba MJ, Pons F, Peiró S, Escrivà M, Muñoz A, Duñach M, de Herreros AG and Baulida J: E-cadherin controls beta-catenin and NF-kappaB transcriptional activity in mesenchymal gene expression. J Cell Sci 121: 2224-2234, 2008.

39. Benoit YD, Guezguez B, Boyd AL and Bhatia M: Molecular Pathways: Epigenetic modulation of wnt/glycogen synthase kinase-3 signaling to target human cancer stem cells. Clin Cancer Res 20: 5372-5378, 2014. 